\title{
Correction to: Holobionts and the ecology of organisms: Multi-species communities or integrated individuals?
}

\section{Derek Skillings ${ }^{1,2}$}

Published online: 22 August 2018

(c) Springer Nature B.V. 2018

\section{Correction to: Biol Philos (2016) 31:875-892 https://doi.org/10.1007/s10539-016-9544-0}

In the original publication, the acknowledgment was published incorrectly. The correct version is given below.

Acknowledgments This paper found its impetus in conversations with Austin Booth, and many of the ideas were worked out in discussion with him. I also thank Peter Godfrey-Smith, Jessie McCormack, Maureen O'Malley, Thomas Pradeu, the participants of the 2015 Philosophy of Biology at Dolphin Beach workshop, and three anonymous reviewers for their help and useful comments. I have received funding from the European Research Council (ERC) under the European Union's Horizon 2020 research and innovation programme-Grant Agreement No. 637647-IDEM.

The original article can be found online at https://doi.org/10.1007/s10539-016-9544-0.

Derek Skillings

derek.skillings@gmail.com

1 Philosophy Program, The Graduate Center, CUNY, 365 Fifth Avenue, Room 7113, New York, NY 10016, USA

2 University of Bordeaux/CNRS, UMR5164, 146 rue Léo Saignat, 33076 Bordeaux, France 